\title{
CHALLENGES AND STRATEGIES USED TO INCREASE THE REPORT OF BRAZILIAN HEMATOPOIETIC STEM CELL TRANSPLANTATION (HSCT) DATA TO THE CENTER FOR INTERNATIONAL BLOOD AND MARROW TRANSPLANT RESEARCH (CIBMTR)
}

\author{
${ }^{1}$ Cinthya Corrêa da Silva, ${ }^{2}$ Heliz Regina Alves das Neves, ${ }^{3}$ Anderson João Simione, ${ }^{4}$ Bruna Letícia \\ da Silva Santos Geraldo, ${ }^{5}$ Eliana Cristina Martins Miranda, ${ }^{6}$ Marcelo C. Pasquini, ${ }^{7}$ Monique Suzanne \\ Ammi, ${ }^{8,9}$ Adriana Seber, ${ }^{9}$ Carmen Silvia Vieitas Vergueiro, ${ }^{10}$ Fernando Barroso Duarte, ${ }^{3}$ Vergilio \\ Antonio Rensi Colturato, ${ }^{1}$ Nelson Hamerschlak
}

${ }^{1}$ Hospital Israelita Albert Eisntein, São Paulo ${ }^{2}$ Hospital de Clínicas - Federal University of Paraná, Curitiba ${ }^{3}$ Amaral Carvalho Hospital, Jau ${ }^{4}$ Bio Sana's, Hospital Sao Camilo, São Paulo ${ }^{5}$ Hematology and Hemotherapy Center, State University of Campinas, Campinas ${ }^{6}$ Clinical Trials Department of Medicine Center for International Blood and Marrow Transplant Research (CIBMTR), Medical College of Wisconsin ${ }^{7}$ Minnesota State University Moorhead, NMDP/Be The Mach, ${ }^{8}$ Hospital Samaritano, São Paulo, ${ }^{9}$ São Paulo State Bone Marrow Association (AMEO), São Paulo ${ }^{10}$ Walter Cantídeo University Hospital, Fortaleza

Correspondence to: Cinthya Corrêa da Silva - cinthya.silva@einstein.br

\begin{abstract}
To increase the report of Brazilian hematopoietic stem cell transplantation (HSCT) data to the Center for International Blood and Marrow Transplant Research (CIBMTR), the Data Managers Working Group (GTGD) of the Brazilian Society of Bone Marrow Transplants (SBTMO), and the Sao Paulo State Bone Marrow Association (AMEO) developed several strategies since 2016: training data managers (GDs) in national and international HSCT meetings, the development of a free online teaching course (EAD) in Portuguese on Transplant Essential Data (TED), online and presential training course for new data managers offered by AMEO, the approval by the National Committee of Ethics in Research (CONEP) of a national multicenter protocol to formalize sharing data of Brazilian transplants with the CIBMTR, and the first multicenter evaluation our HSCT results using the CIBMTR Data Back to Center. The contract between SBTMO and CIBMTR was signed in 2019 and GTGD of the SBTMO was officially created. These actions resulted in an increase from 24 to 41 transplant centers registered at the CIBMTR from 2016 to 2019. The process of increasing adherence and continuity of HSCT reports to the CIBMTR is complex and requires commitment of all professionals involved HSCT. The success of this process depends on education of the GD and the involvement of all HSCT directors.
\end{abstract}

Keywords: Database. Hematopoietic Stem Cell Transplantation. Information system.

\section{INTRODUCTION}

Hematopoietic stem cell transplants (HSCT) are used to treat many onco-hematological and benign diseases 1 . For many patients, it is the only treatment option that offers potential for curing their disease, 2 as well as offering quality of life. According to the estimate of the Brazilian Society of Bone Marrow Transplantation (SBTMO) and the records of the Brazilian Association of Organ Transplantation (ABTO), about $37,000,000$ procedures were performed in the country from 1979 to 20193. The Brazilian Transplant Registry (RBT), ran by the ABTO, provides some quantitative indicators and survival data. In 2019, 3,805 transplants were reported: 1,428 allogeneic and 2,377 autologous4. However, in Brazil, there is not any specific and consolidated HSCT registry. Many Brazilian centers do not have electronic infor- 
mation system and/or medical records that meet their needs, and those who do have such tools, often do not have their data organized in a standardized and integrated way, what may make difficult or impossible to analyze many indicators, such as outcomes and transplant-related complications, multicenter studies, and benchmarking.

However, there are registries developed and made available globally, such as the Center for International Blood and Marrow Transplant Research (CIBMTR), a North American platform created in 2004, merging the International Bone Transplant Transplant Registry (IBMTR) and the National Marrow Donor Program (NMDP)5. The CIBMTR offers an online platform, where centers performing HSCT and/or cell therapy worldwide can insert their data and retrieve relevant data for multicenter studies or for the center, including self-evaluation and benchmarking. Therefore, the objective of this manuscript is to describe the challenges, strategies and results obtained since 2016 with the GTGD, AMEO and SBTMO collaboration to expand and improve the inclusion of Brazilian transplant centers to the CIBMTR.

\section{METHODOLOGY}

Brazil's relationship with the CIBMTR begun with the affiliation of the Hospital de Clínicas - Universidade Federal do Paraná (HC-UFPR) to the former IBMTR, in the 1980s, before NMDP and IBMTR formed the CIBMTR. After that, other Brazilian centers joined the CIBMTR, but Brazilian data entry varied over time (Figure 1).

In 2016, a partnership between HC-UFPR, Hospital Amaral Carvalho (HAC) and Hospital Israelita Albert Einstein (HIAE) originated the data managers' working group (GTGD). Subsequently, in 2018, the Bone Marrow Association of the State of São Paulo (AMEO) developed an online training course for new data managers (GD) working at centers authorized to perform Unrelated Donor Transplants. This program, funded by the Brazilian Government (National Program to Support Oncological Attention - Pronon), included a scholarship to the Data Managers and a notebook for programs at public transplant centers. Tools developed by two transplant centers using Access and REDCap to capture all CIBMTR data fields and enable later filling of the online CIBMTR forms were shared to all interested institutions. The development of instruments and strategies to improve adherence to reporting to the CIBMTR has been gradually implemented and important changes are foreseen in the area of HSCT. The GTGD is consolidat- ed and the mission, vision and values of the group were established (Figure 2).

\section{RESULTS}

Recognizing the importance of the CIBMTR, several initiatives were developed to train Brazilian professionals with support from the CIBMTR: consecutive visits to the CIBMTR were performed, the first in October 1996 by the GD from the HC-UFPR, then, in February 2009, GD from UNICAMP, in March 2016, GDs from HAC and HIAE, and in 2019, GDs from Biosana's and Ameo. The 2016's visit resulted in a partnership between SBTMO and CIBMTR that offered the first Brazilian GD meeting at the annual meeting of the SBTMO, with approximately 15 participants. This GD meeting was repeated annually with support from the CIBMTR and the number of participants gradually increased, reaching 52 participants from 29 centers in 2019.

Since first Meeting of GDs in 2016, a voluntary work of the GDs from HC-UFPR, HAC and HIAE started. Also, in 2016 the GDs created the first Brazilian online training for filling in the CIBMTR forms. This EAD tool was made available free of charge to all HSCT centers in Brazil; 65,535 people accessed the tutorial, and 573 completed the pre-TED training (Form 2400) and 202 completed the post-TED training (Form 2450). The result of this training was presented at the BMT Tandem Meeting in 2017 and received the award for best work in the GDs category. [6]

In 2017, the HIAE Research Ethics Committee (CEP) approved a multicenter trial submitting data to the CIBMTR, entitled "Multicenter Registry of Autologous and Allogeneic Hematopoietic Stem Cell Transplants (HCT) for malignant and non-malignant diseases performed in Brazil and reported at the Center for International Blood and Transplant Research (CIBMTR)". With this approval, it was possible to make some analyses, with the return of the CIBMTR database, through a business intelligence tool $(\mathrm{BI})$, the Data Back to Center (DBtc). Through this tool provided by CIBMTR, it was possible to extract a large volume of data in Excel format, ready for analysis, in a short period of time. The analysis was made joining the spreadsheets extracted from the DBtC by each of the 7 participating centers and, even with a modest number of centers, there was an expressive number of transplants. The HAC GD unified the worksheets and analyzed the data using the SPSS software (version 15.0 for windows). Patients undergoing the 1st HSCT from 2008 to 2018, a total of 3,994 patients, were included. This analysis showed the diseases most fre- 
quently transplanted in Brazil, as well as the increasing number of transplants from HLA mismatched related donors in recent years; stem cell sources and overall survival (SG) by diagnoses were described for adult and pediatric patients [7]. This analysis resulted in two abstracts selected for oral presentation at the Tandem Meetings and SBTMO Annual Meeting in 2019. In the latter, the GDs received the "Young Scientist Award - Dr.Ricardo Pasquini".

In 2019, this project was approved by the National Research Commission (CONEP), the Brazilian Central IRB. Today we have 20 participating centers and eight more are being included. Although the number of participating centers is modest, they represent a significant part of the transplants performed in the country.

Over time, the actions of these DGs have highlighted the fundamental importance of this profession in the HSCT scenario and, although it is not formally recognized in the country, SBTMO officialized the creation of the GTGD in 2019 to continue and further expand the participation in national and international registries (ABTO, WBMT/LABMT and CIBMTR).

To effectively start the work, the GTGD created a small executive committee: Anderson João Simione from HAC as president, Cinthya Corrêa da Silva from HIAE as vice-president, and Heliz Regina Alves das Neves from HC-UFPR as scientific coordinator. The identity of the group was defined, establishing its mission, vision and value, in addition to the elaboration of a logo (in Figure 2 ). Immediately thereafter, with the need of more professionals to contribute, Bruna Leticia S. S. Geraldo from Bio Sana's - IBCC was added for administrative and scientific support and Monique S. Ammi, as a representative from the CIBMTR.

Currently there are monthly meetings promoted by GTGD, where issues are addressed in the area of HSCT by the GDs themselves and expert guests.

Other initiatives were added to the actions in the preparation and consolidation of the Brazilian GDs, such as the start of the data manager training project in 2018, by AMEO. In 2019, AMEO and the GD of Bio Sana's visited the CIBMTR and received specific training to fill in the forms and train the professionals. AMEO, in partnership and financial support from the Brazilian government through the National Program for Support to Oncologic Care (PRONON) has developed an innovative strategy to empower and encourage new GD in the country. Of the $36 \mathrm{HSCT}$ centers perform unrelated donor transplants, 30 participated in a 14-month training for new GDs. The program provided notebooks and financial support to the participants. The training was performed with online classes three times a week in three cycles, followed by a three-day visit to each center by one of the two AMEO nursing instructors. Of the total institutions participating in this training, 57\% were public and $83 \%$ of the new GDs received financial assistance, $60 \%$ of whom were TCTH nurses. In addition, $90 \%$ of the new GDs completed the first of the three modules with a frequency above $75 \%$. According to the new GDs evaluation, the program is excellent and of high importance to $100 \%$ of them.

These actions resulted in an increase from 24 to 41 transplant centers registered at the CIBMTR from 2016 to 2019 (Figure 1). Actually, there are 32 Brazilian transplant centers reporting data to CIBMTR (Table 1).

In 2019, the SBTMO signed a contract of partnership with the CIBMTR to have a HSCT registry with good quality and accuracy of data that are necessary to generate indicators and outcomes of HSCT performed in Brazil.

\section{DISCUSSION}

The issue behind all the above described initiatives is the lack of a national outcomes registry that may allow data analyses and multicenter studies. The process of developing a system for the HSCT is complex, as it requires planning, investment, infrastructure, time, professional training, awareness of transplant teams and support from government entities. The CIBMTR offers many tools such as QlikView free of charge, in addition to the system for data entry, which enables data analysis. However, there are some limiting factors of this toll, such as the impossibility of overall survival analysis comparing more than two groups.

Another benefit of reporting to the CIBMTR is the use of the data devolution tool, the DBtC, where each center can extract spreadsheets with its data and develop analyses through other statistical software. CIBMTR also supports GDs, such as content for guidance on filling in forms, help desk service, online question shift (ServiceNow). In addition, centers registered as research have a refund after filling out Comprehensive Research Forms (CRF), what can help to finance data management, and there is also a scholarship to non-U.S. GDs to participate in the annual TCT Meeting.

Through the approval by the Ethics Committee of the multicenter trial to report to the CIBMTR made it possible to legally send data to North America and 
made it easier to new centers to join. The first Brazilian multicenter study using the CIBMTR database, demonstrated the effectiveness of $\mathrm{BI}$ tools, used to have the center data and analyze it, DBtC and QlikView, respectively. The use of these tools allowed an analysis, in a short period of time, and to have relevant results from Brazilian transplant centers.

There are some limitations when using DBtC, as incomplete data retrieval, lack of information on relapse, the categorization of haploidentical donors, and the delay to have data from the CIBMTR portal, which is not updated in real time. However, CIBMTR is receptive to discuss problems brought by the Brazilian teams and to help finding solutions.

The training in the CIBMTR of the GDs brought new perspectives to the professionals, because in addition to learning, demonstrated the importance of the category for the HSCT, as already seen in the USA. The education of this professional, either through fast courses (EAD) or intensive training, as promoted by AMEO, decreased the gap between different professionals (nurses, biomedicals, system analysts, secretaries, and others) and brought the GDs closer to each other as a group. Since the recognition of the GTGD by the SBTMO, their work has been officialized and their responsibility has increased in gathering HSCT data for the county and designing future guidelines. The agreement signed between SBTMO and CIBMTR formalized the use of Registry, promoting greater adherence of the centers in sending data. Currently, the interface of the system is in English and the translation and adaptation to the Brazilian reality is being discussed, as happened with Canada and Japan.

\section{CONCLUSION}

Our initiatives have yield positive results, such as the better qualification of the professionals and the increasing number of centers affiliated to CIBMTR. There is still much to be done. Now, one should continue and improve the qualification of the GDs and maintain the commitment of the HSCT centers to include new patients and complete their long-term follow-up.
Next, to have support from HSCT centers and government to provide infrastructure, training and awareness of the multidisciplinary team to this activity. The future challenges are the development of the SBTMO website about data management, including support (already under construction), the Continuous Process Improvement (CPI) infrastructure to ensure the quality of data from affiliated centers, the creation of a commission for the organization and regulation of scientific production, and many other projects.

It is clear to us that reaching most of the affiliated and active centers we will be able to better understand the Brazilian HSCT scenario. Afterall, research based on data captured with quality, accuracy and security, it is possible to enable multicenter studies, benchmarking and, consequently, improve the care of patients undergoing HSCT.

\section{ACKNOWLEDGEMENTS}

GTGD is in debt with:

To Dr. Ricardo Pasquini, one of the pioneers in bone marrow transplantation in Latin America.

To SBTMO for the support provided to data managers (GD) in Brazil, through different incentives, especially during the officialization of the working group via The Society.

To Dr. Nelson Hamerschlak, a visionary physician who encouraged this movement in the country, from 2016.

To Dr. Marcelo Pasquini who enables direct contact with CIBMTR and brings updates and teachings from the research record.

To all multidisciplinary teams of TMO that directly or indirectly enabled the development of the work of the GDs.

Patients who undergo this treatment modality and contribute to scientific research by making their data available. 


\section{REFERENCES}

1. Majhail NS, et tal. Indications for Autologous and Allogeneic Hematopoietic Cell Transplantation: Guidelines from the American Society for Blood and Marrow Transplantation. Biology Blood Marrow Transplant. Blood M 2015 Nov; 21(11): 18631869. doi: 10.1016/j.bbmt.2015.07.032. Epub 2015 Aug 7

2. Copelan EA. Hematopoietic stem-cell transplantation. N Engl J Med. 2006;354(17):1813-26

3. Brazilian Society of Bone Marrow Transplantation. SBTMO participates in INCA's TCTH Update Journey in June. Available in: http://www.sbtmo.org. br/noticia.php?id=417. Accessed 2017 (Sep 29).

4. Brazilian Association of Organ Transplantation [internet page], [Accessed on 05/21/2020], Available at: http://www.abto.org.br/abtov03/Upload/file/ RBT/2019/RBT-2019-leitura.pdf
5. Center for International Blood and Marrow Transplant Research [internet page] [Accessed 21/05/2020]. Available in: https://www.cibmtr. org/About/WhoWeAre/History/Pages/index.aspx

6. Center for International Blood and Marrow Transplant Research [website] [Accessed 21/05/2020]. Available in: https://www.cibmtr.org/Meetings/ Materials/Documents/Oral\%20Abstract\%20\%20 Cinthya\%20da\%20Silva\%20(02\%2007\%202017). pdf.

7. Center for International Blood and Marrow Transplant Research [website] [Accessed 26/05/2020]. Available in: https://www.cibmtr.org/Meetings/ Materials/CRPDMC/_layouts/15/WopiFrame.aspx?sourcedoc=/Meetings/Materials/CRPDMC/Documents/2019/TCT\%202019\%20Cinthya\%20Anderson\%20abst\%20slides.pptx\&action=default

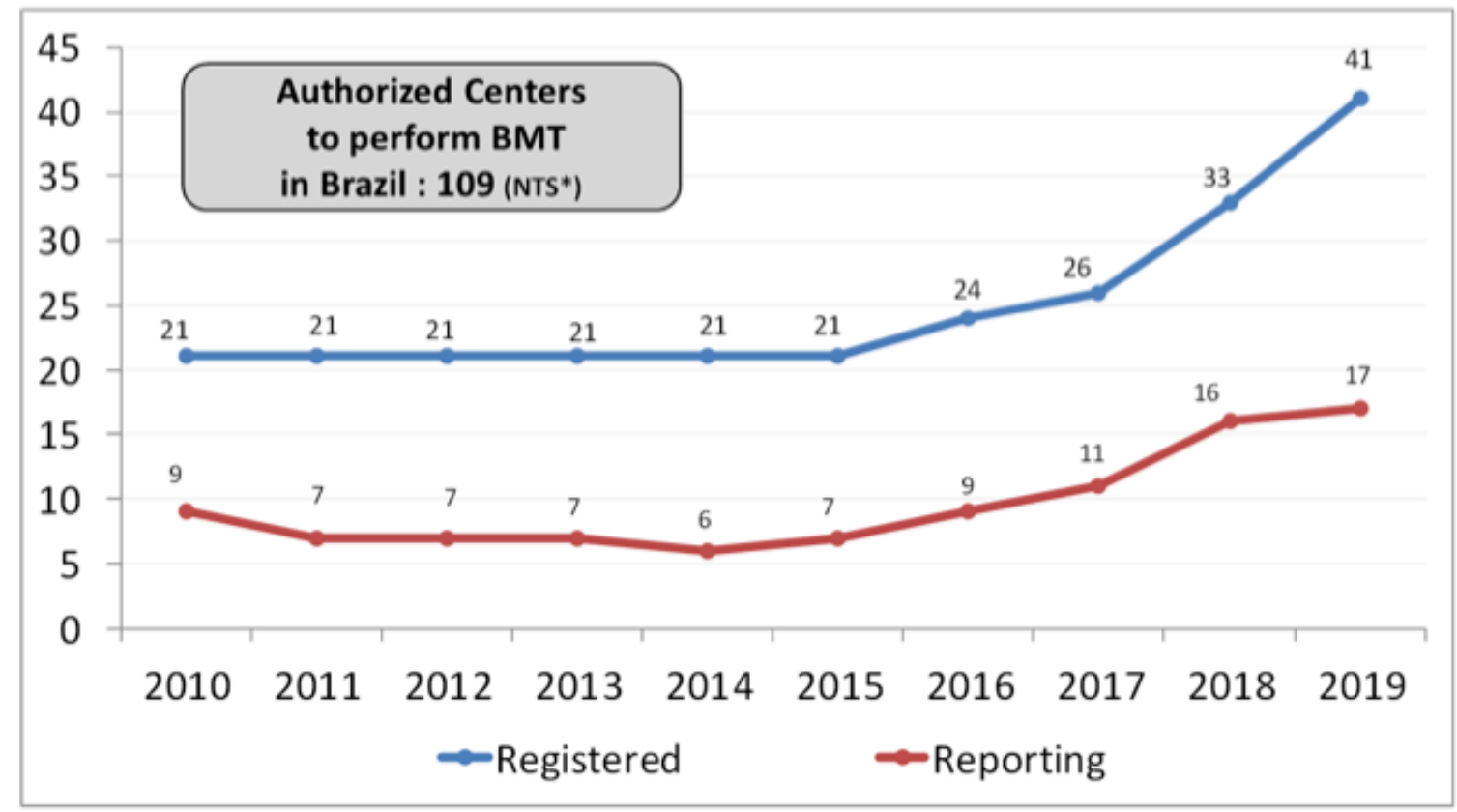

${ }^{\star}$ National Transplant System

(source: INFOREQ\#2001-02, CIBMTR)

Figure 1 - Number of Centers Registered and Actively Reporting to the CIBMTR 
Table 1 - Brazilian transplant centers that currently report data to CIBMTR

\begin{tabular}{|c|c|c|}
\hline NAME & CITY & COUNTRY \\
\hline Hospital Nossa Senhora das Graças - IP & Curitiba & Brazil \\
\hline Hospital Nossa Senhora das Graças - IF & Curitiba & Brazil \\
\hline Universidade Federal de São Paulo - Hospital São Paulo & São Paulo & Brazil \\
\hline Hospital e Maternidade Brasil & Santo André & Brazil \\
\hline Associação Hospitalar Moinhos de Vento & Porto Alegre & Brazil \\
\hline Bio Sana's São Camilo & São Paulo & Brazil \\
\hline A.C. Camargo Cancer Center & São Paulo & Brazil \\
\hline UNICAMP - HEMOCENTRO & Campinas & Brazil \\
\hline Hospital Amaral Carvalho & Jau & Brazil \\
\hline UFMG Hospital das Clínicas Servico de Transplante de Medula Óssea & Belo Horizonte & Brazil \\
\hline Hospital Leforte Liberdade & São Paulo & Brazil \\
\hline Hospital Erasto Gaertner & Curitiba & Brazil \\
\hline Hospital de Clínicas de Porto Alegre & Porto Alegre & Brazil \\
\hline Instituto de Oncologia Pediátrica - GRAACC & São Paulo & Brazil \\
\hline $\begin{array}{c}\text { Instituto de Cardiologia do Distrito Federal - Unidade de TMO Pietro } \\
\text { Albuquerque }\end{array}$ & Brasilia & Brazil \\
\hline Natal Hospital Center & Natal & Brazil \\
\hline Hospital Universitario da Universidade Federal de Juiz de Fora & Juiz de Fora & Brazil \\
\hline $\begin{array}{c}\text { Instituto da Criança - Hospital das Clínicas da Faculdade de Medicina } \\
\text { da Universidade de São Paulo (ITACI) }\end{array}$ & São Paolo & Brazil \\
\hline Instituto Nacional de Câncer & Rio de Janeiro & Brazil \\
\hline Hospital de Clínicas - UFPR & Curitiba & Brazil \\
\hline Fundação Pio XII - Hospital de Câncer de Barretos & Barretos & Brazil \\
\hline Hospital Samaritano & São Paulo & Brazil \\
\hline Albert Einstein Hospital & São Paulo & Brazil \\
\hline Hospital Sírio Libanês & São Paulo & Brazil \\
\hline Hospital São Camilo & São Paulo & Brazil \\
\hline Federal University of Ceará & Fortaleza & Brazil \\
\hline Complexo Hospitalar de Niterói & Niterói & Brazil \\
\hline Centro de Pesquisas Oncológicas Dr. Alfredo Daura Jorge (CEPON) & Florianopolis & Brazil \\
\hline IBCC - Instituto Brasileiro de Controle do Câncer & São Paulo & Brazil \\
\hline CTMO-HCFMUSP & São Paulo & Brazil \\
\hline Real e Benemérita Sociedade de Beneficiência Portuguesa de São Paulo & São Paulo & Brazil \\
\hline Hospital Universitario Clementino Fraga Filho, Univ. Fed. RJ & Rio de Janeiro & Brazil \\
\hline
\end{tabular}




\section{Values Statement}

\section{Mission}

\section{Vision}

\section{Values}

- Provide support to Brazilian data managers in collecting, managing, and analyzing information from patients undergoing hematopoietic stem cell transplantation (HSCT) and cell therapies.

- To be a leader and to point out solutions and strategies to promote the improvement of data collection, ensuring efficiency, reliability and security of information, contributing to scientific production and, consequently, to the health conditions of transplanted patients.

Motivation: Guarantee the quality and confidentiality of HSCT data;

- Commitment: Ethics, solidarity, respect, health, faith and love;

- Groundwork : Responsibility, continuous learning and team spirit.

Source: https://www.cibmtr.org/Meetings/Materials/CRPDMC/Pages/2020-Clinical-Research-Professionals--Data-Management.aspx Source: https://www.cibmtr.org/About/WhoWeAre/Centers/Pages/index.aspx?country=Brazil

Figure 2 - Statement Values: Mission, Vision and Values

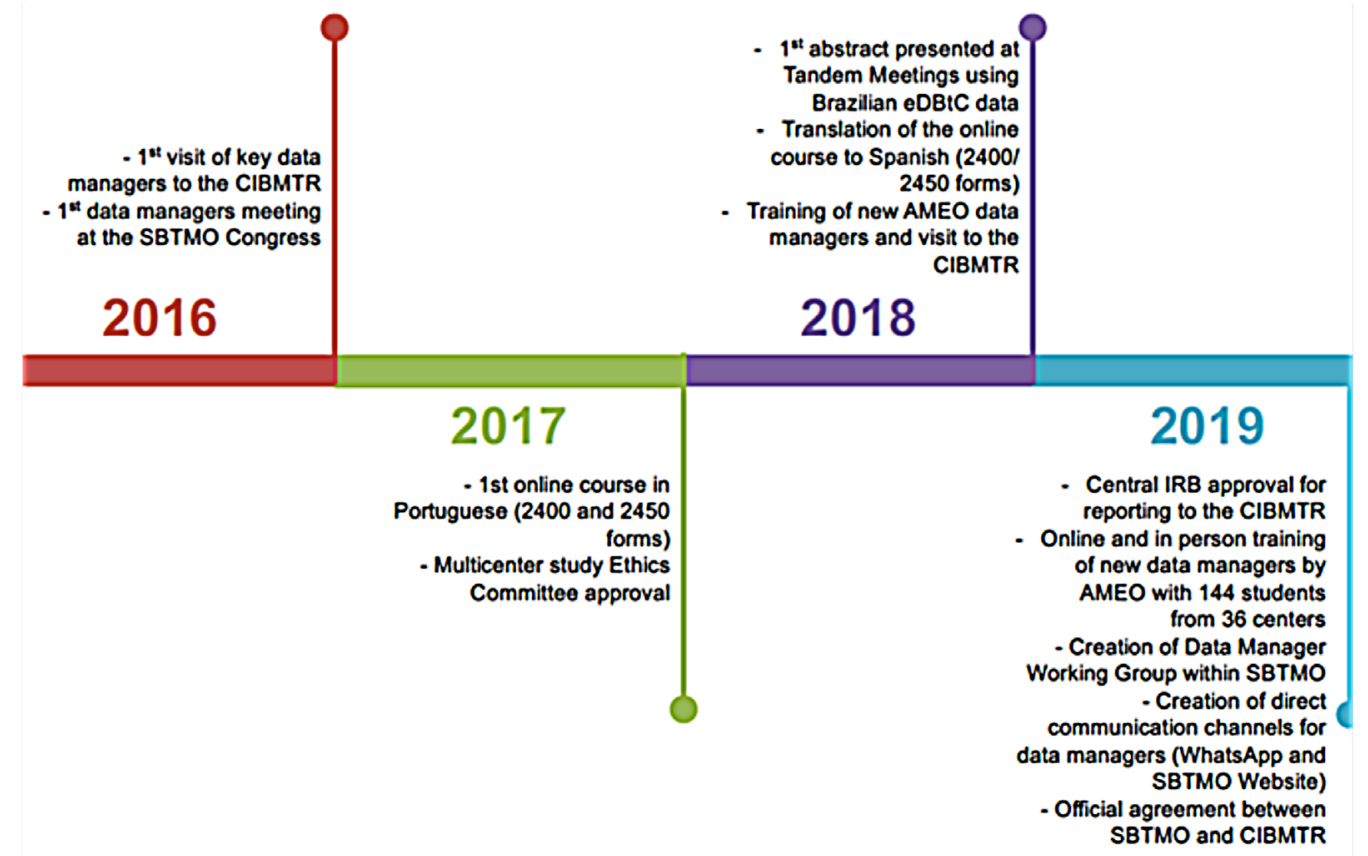

Source: https://www.cibmtr.org/Meetings/Materials/CRPDMC/Pages/2020-Clinical-Research-Professionals--Data-Management.aspx

Figure 3 - Methods: Timeline of Actions, 2016 - 2019 